\title{
Overexpression of COX-2 but not indoleamine 2,3-dioxygenase-1 enhances the immunosuppressive ability of human umbilical cord-derived mesenchymal stem cells
}

\author{
DONG LI, YAN HAN, YONG ZHUANG, JINQIU FU, HUAN LIU, QING SHI and XIULI JU \\ Cryomedicine Laboratory, Qilu Hospital, Shandong University, Ji'nan, Shandong 250012, P.R. China
}

Received June 24, 2014; Accepted March 5, 2015

DOI: $10.3892 / \mathrm{ijmm} .2015 .2137$

\begin{abstract}
Owing to their immunosuppressive properties mesenchymal stem cells (MSCs) are widely applicable in the treatment of autoimmune disease. The aim of this study was to investigate whether the indoleamine 2,3-dioxygenase-1 $(I D O-1)$ and cyclooxygenase-2 $(C O X-2)$ genes enhanced the immunosuppressive functional ability of MSCs following stable transfection. To strengthen the immunomodulatory ability of MSCs, $I D O-1$ and $C O X-2$ were overexpressed in umbilical cord progenitor cell-derived MSCs using recombinant plasmids and electroporation. RT-qPCR analysis and western blotting confirmed the expression of IDO-1 and COX-2 in transfected MSCs. Further functional assays in co-culture experiments, including lymphocyte proliferation and cytotoxicity assays showed that COX-2-transfected MSCs possessed more potent immunomodulatory cells than the untreated MSCs, or MSCs transfected with IDO-1. Additionally, synthesis of interferon- $\gamma$ and tumor necrosis factor- $\alpha$ (TNF- $\alpha$ ) was significantly inhibited in lymphocytes co-cultured with COX-2-transfected MSCs, which was consistent with changes in immune-related genes in MSCs. An enhanced expression of IDO-1, COX-2, heme-oxygenase-1, inducible nitric-oxide synthase, TNF- $\alpha$-stimulated gene/protein- 6 , transforming growth factor- $\beta$ (TGF- $\beta$ ), human leukocyte antigen molecule 5 (HLA-G5) and interleukin-10 (IL-10) was identified following COX-2 transfection. We showed that the overexpression of COX-2 enhanced the immunosuppressive function of MSCs. COX-2-modified MSCs more potently inhibited the activation and proliferation of peripheral blood mononuclear cells.
\end{abstract}

\section{Introduction}

Mesenchymal stem cells (MSCs) are of stromal origin and can be isolated from various human tissues, including bone

Correspondence to: Dr Xiuli Ju, Cryomedicine Laboratory, Qilu Hospital, Shandong University, 107 Wenhua Xi Road, Ji'nan, Shandong 250012, P.R. China

E-mail: shellysdcn07@gmail.com

Key words: mesenchymal stem cells, transfection, indoleamine 2,3dioxygenase, immunomodulation, cyclooxygenase- 2 marrow, adipose tissue, skeletal muscle, synovium, gingiva, amniotic fluid, cord blood, and the umbilical cord. MSCs are an extremely promising source of adult stem cells that may be used for cell-based therapeutics, due in part to their substantial ability to differentiate into multilineages and broad secretory activities (1-4). Preclinical studies have suggested the potential for using MSCs in the settings of tissue repair in type 1 diabetes (5), acute lung injury (6,7), radiation protection (8) and nephropathy (9).

However, it was suggested that the immunomodulatory activity of MSCs is an important consideration in cell therapy (10). Additionally, in vitro evidence suggests that MSCs directly modulate T-cell function. MSCs inhibit the maturation and migration of various antigen-presenting cells, suppress B-cell activation, induce suppressor T-cell formation, and alter the expression of several receptors necessary for antigen capture and processing $(11,12)$. This immunosuppressive activity of MSCs may also play an important role in the therapy of autoimmune diseases (13), and the prevention of acute graft-versus-host disease (aGVHD) (14).

Previous findings have shown that the recommended infusion of adoptively transferred MSCs ranges from $10^{5} / \mathrm{kg}$ to $10^{7} / \mathrm{kg}$ body weight, and that an association exists between the anticipated therapeutic effect and the increasing quantity of MSCs (15-19). However, infused MSCs are, not only found in the bone marrow and injured tissues, but are also located in the lungs $(20,21)$. The dangers of pulmonary embolism and cardiac dysfunction increase with the number of adoptively transferred input cells (22). Therefore, this study aimed to obtain a new population of MSCs exhibiting more potent immunosuppressive functions with subsequent improvement in their clinical application without the need for an increasing cell dose.

Numerous molecules participate in the immunomodulation process of MSCs, including prostaglandin E2 (PGE2) (23), indoleamine 2,3-dioxygenase (IDO) (24), nitric oxide (25), transforming growth factor- $\beta$ (TGF- $\beta$ ) (26), and interleukin-6 (IL-6) (27). Of these molecules, IDO-1 and cyclooxygenase-2 (COX-2) are key factors in tryptophan catabolism and PGE2 synthesis, respectively. When PGE2 production is inhibited, immunomodulation is weakened $(23,24,28)$. Tryptophan is an essential amino acid in T-cell proliferation, and its depletion or accumulation of its degradation product kynurenine, adversely affect T-cell amplification (24). 
In view of the critical roles of IDO- 1 and COX-2 in immunomodulation, we attempted to upregulate the expression levels of IDO- 1 and COX-2 to enhance the synthesis of PGE2 and kynurenine, and to determine whether the immunosuppressive ability of MSCs can be improved.

\section{Materials and methods}

Culture of MSCs. Umbilical cords were obtained from clinically normal pregnancies after participants provided informed consent. The study was approved by the local ethics committee of Qilu Hospital. Umbilical cords were excised and washed in $0.1 \mathrm{~mol} / 1$ phosphate buffer ( $\mathrm{pH}$ 7.4) to remove residual blood. The cords were dissected, and the blood vessels were removed. The remaining tissues were cut into small sections $\left(1-2 \mathrm{~mm}^{3}\right)$ and placed in tissue culture plates with low-glucose Dulbecco's modified Eagle's medium (DMEM), which was supplemented with $10 \%$ fetal bovine serum (FBS) (Gibco-BRL, Grand Island, NY, USA), $2 \mathrm{ng} / \mathrm{ml}$ vascular endothelial growth factor (VEGF), $2 \mathrm{ng} / \mathrm{ml}$ epidermal growth factor (EGF), $2 \mathrm{ng} / \mathrm{ml}$ fibroblast growth factor (all from R\&D Systems, Minneapolis, MN, USA), $100 \mathrm{U} / \mathrm{ml}$ penicillin, and $100 \mu \mathrm{g} / \mathrm{ml}$ streptomycin (Gibco-BRL). Cultures were maintained at $37^{\circ} \mathrm{C}$ in a fullyhumidified atmosphere with $5 \% \mathrm{CO}_{2}$ in air. The media were changed every 3-4 days. Adherent cells proliferated from individual explanted tissues 7-12 days after the culture was initiated. Subsequently, small tissue specimens were removed from the culture, and the adherent fibroblast-like cells were cultured to confluence for 2-3 weeks. The cells were trypsinized using $0.25 \%$ trypsin (Gibco-BRL) and then passaged at a density of $1 \times 10^{4}$ cells $/ \mathrm{ml}$ in the culture medium exactly as described above. The cells were used after 5 or more passages.

Cell surface antigen phenotyping. Fifth- to seventh-passage cells were collected and treated with $0.25 \%$ trypsin. The cells were then stained with either fluorescein isothiocyanate (FITC)-conjugated or phycoerythrin (PE)-conjugated monoclonal antibodies in $100 \mu \mathrm{l}$ phosphate buffer for $15 \mathrm{~min}$ at room temperature, as per the manufacturer's instructions. The antibodies used in this assay were targeted against human cell-surface expressed antigens including CD29, CD34, CD31, CD44, CD45, CD73, CD90 and CD105 (SeroTec, Raleigh, NC, USA). The cells were analyzed by flow cytometry (Guava easyCyte 6HT-2L; Merck Millipore, Billerica, MA, USA). Positive cells were counted and compared with the signal of the corresponding immunoglobulin isotypic controls.

Recombinant plasmid construction and transfection. For construction of the expression plasmid, the IDO-1 and the COX-2 inserts were isolated by polymerase chain reaction (PCR) amplification from a complementary DNA (cDNA) library (GeneChem, Shanghai, China) and digested with the restriction endonucleases EcoRI and BglII (MBI Fermentas, Burlington, ON, Canada). The inserts were subsequently linked to the pEGFP-N3 expression plasmids (Bio-Asia Co., Shanghai, China) with T4 DNA ligase (TransGen, Beijing, China). The ligation products were transformed into competent Escherichia coli DH5 $\alpha$ (TransGen) and then selected using the kanamycin resistance method. Recombinant plasmids were sequenced (ABI Prism 3100 DNA Sequencer; Applied
Biosystems, Foster City, CA, USA) and confirmed to contain the entire coding sequence of IDO-1 or COX-2. Clones with the correct sequence were amplified for further transfection. The new recombinant plasmids were designated as pEGFP-IDO-1 and pEGFP-COX-2.

The recombinant plasmids were transfected into MSCs with a gene transfection instrument (SCIENTZ-2C; Scientz Co., Ningbo, China) and electroporation cuvettes (Bio-Rad, Hercules, CA, USA). A successful transduction was confirmed by visualizing the expression of enhanced green fluorescent protein (EGFP; included in the pEGFP-N3 vector) after 4 days of culture. The cells were maintained and allowed to grow for another 3-5 days, and the expression level of IDO-1 and COX-2 was confirmed by western immunoblotting as described below.

The experiments were divided into four groups: i) untreated MSCs were the control group, ii) cells transfected with only the IDO-1 gene were the MSCs (IDO-1) group, iii) cells transfected with only the $C O X-2$ gene were the MSCs (COX-2) group, and iv) cells described as the MSCs (IDO-1/COX-2) group, were simultaneously transfected with the IDO-1 and COX-2 plasmids. The expression levels of the transferred plasmids were the same in each group.

Protein extraction and western immunoblotting. To verify the expression levels of IDO-1 and COX-2, the transfected cells were collected and resuspended in protein lysis buffer (RIPA: PMSF at a ratio of 100:1; Solarbio, Beijing, China) according to the manufacturer's instructions. Lysates were incubated on ice for $30 \mathrm{~min}$ and then centrifuged at $12,000 \mathrm{rpm}$ and $4^{\circ} \mathrm{C}$ for $20 \mathrm{~min}$. Equal amounts of proteins (15 $\mu \mathrm{g}$ for each sample) were separated by sodium dodecyl sulfate-polyacrylamide gel electrophoresis (SDS-PAGE) and then transferred to polyvinylidene difluoride (PVDF) membranes. The membranes were incubated with antibodies against IDO-1, COX-2 or $\beta$-actin (Abcam Inc., Cambridge, MA, USA) at $4^{\circ} \mathrm{C}$ overnight. Antibody binding was assessed by incubation with horseradish peroxidase-conjugated secondary antibodies (Beyotime, Shanghai, China). Chemiluminescence was detected using an ECL Plus immunoblotting detection system (Beyotime).

Reverse transcription-quantitative PCR (RT-qPCR) assay of immune-regulated gene $m R N A$ expression. In order to detect changes in immune-related genes in transfected MSCs, total RNA was extracted using TRIzol reagent (Invitrogen, Carlsbad, CA, USA) following the manufacturer's instructions. Total RNA was reverse transcribed to cDNA with the Omniscript cDNA Synthesis kit (Qiagen, Hamburg, Germany) according to the manufacturer's instructions. In a typical procedure, $0.2 \mu \mathrm{g}$ of total RNA was reverse transcribed in a final volume of $20 \mu \mathrm{l}$ containing the following: $1 \mathrm{X} \mathrm{RT}$ buffer, deoxynucleotide triphosphate mix (5 $\mathrm{mM}$ each), RNase inhibitor (10 U/ $\mu 1$ RNase out; Invitrogen), oligo(dT) primers and 4 units Omniscript RT. The samples were incubated at $37^{\circ} \mathrm{C}$ for $60 \mathrm{~min}$, and the resulting cDNA was stored at $-80^{\circ} \mathrm{C}$ until RT-qPCR analysis was initiated using an ABI 7500 PCR system and SYBR-Green I dye (Toyobo, Osaka, Japan). The primers used are provided in Table I. The reagents and primers were obtained from Bioasi Co., Ltd., Shanghai, China. $\beta$-actin was used as an internal control. The expression of each gene 
Table I. Primer sequences used in PCR.

\begin{tabular}{|c|c|c|}
\hline Genes & Primer sequences & Size (bp) \\
\hline$I D O-1^{\mathrm{a}}$ & $\begin{array}{l}\text { 5'-GGAAGATCTTCCATGGCACACGCTATGGAAAAC } \\
\text { 5'-CCGGAATTCCGGACCTTCCTTCAAAAGGGATTTC }\end{array}$ & 1,209 \\
\hline$C O X-2^{\mathrm{a}}$ & $\begin{array}{l}\text { 5'-GGAAGATCTTCCATGCTCGCCCGCGCCCTGCTGC } \\
\text { 5'-CCGGAATTCCGGCAGTTCAGTCGAACGTTCTTTTAG }\end{array}$ & 1,812 \\
\hline$I D O-1$ & $\begin{array}{l}\text { 5'-GCCCTTCAAGTGTTTCACCAA } \\
\text { 5'-GCCTTTCCAGCCAGACAAATAT }\end{array}$ & 90 \\
\hline$C O X-2$ & $\begin{array}{l}\text { 5'-GGTCTGGTGCCTGGTCTGAT } \\
\text { 5'-TCCTGTTTAAGCACATCGCATACT }\end{array}$ & 80 \\
\hline$H M O X-1$ & $\begin{array}{l}\text { 5'-AGGGAAGCCCCCACTCAAC } \\
\text { 5'-ACTGTCGCCACCAGAAAGCT }\end{array}$ & 81 \\
\hline$I L-2$ & $\begin{array}{l}\text { 5'-CCAGGATGCTCACATTTAAGTTTTAC } \\
\text { 5'-GAGGTTTGAGTTCTTCTTCTAGACACTGA }\end{array}$ & 87 \\
\hline$I L-10$ & $\begin{array}{l}\text { 5'-GCCTTGTCTGAGATGATCCAGTT } \\
\text { 5'-TCACATGCGCCTTGATGTCT }\end{array}$ & 85 \\
\hline iNOS & $\begin{array}{l}\text { 5'-GGTGGAAGCGGTAACAAAGG } \\
\text { 5'-TGCTTGGTGGCGAAGATGA }\end{array}$ & 81 \\
\hline$T G F-\beta 1$ & $\begin{array}{l}\text { 5'-GGGAAATTGAGGGCTTTCG } \\
\text { 5'-GAACCCGTTGATGTCCACTTG }\end{array}$ & 83 \\
\hline$I L-1 \alpha$ & $\begin{array}{l}\text { 5'-GACGCCCTCAATCAAAGTATAATTC } \\
\text { 5'-TCAAATTTCACTGCTTCATCCAGAT }\end{array}$ & 89 \\
\hline$I L-1 \beta$ & $\begin{array}{l}\text { 5'-GCGGCATCCAGCTACGAAT } \\
\text { 5'-GTCCATGGCCACAACAACTG }\end{array}$ & 80 \\
\hline$I F N-\gamma$ & $\begin{array}{l}\text { 5'-CCAACGCAAAGCAATACATGA } \\
\text { 5'-TTTTCGCTTCCCTGTTTTAGCT }\end{array}$ & 72 \\
\hline$\beta$-actin & $\begin{array}{l}\text { 5'-GGACATCCGCAAAGACCTGTA } \\
\text { 5'-GCATCCTGTCGGCAATGC }\end{array}$ & 80 \\
\hline
\end{tabular}

${ }^{\text {aPrimers }}$ of IDO-1 and COX-2 used to obtain complete CDS of genes by polymerase chain reaction. PCR, polymerase chain reaction; IDO-1, indoleamine 2,3-dioxygenase-1; COX-2, cyclooxygenase-2; IL-2, interleukin-2; iNOS, inducible nitric-oxide synthase; TGF- $\beta 1$, transforming growth factor- $\beta$; IFN- $\gamma$, interferon- $\gamma$.

was determined using the $2^{-\Delta \Delta \mathrm{CT}}$ method. The RT-qPCR conditions used were: 1 cycle at $95^{\circ} \mathrm{C}$ for 4 min followed by $94^{\circ} \mathrm{C}$ for $15 \mathrm{sec}$ at $60^{\circ} \mathrm{C}$ for $1 \mathrm{~min}$, and for a total of 40 cycles. Data were analyzed using Sequence Detection software version 1.4 (Applied Biosystems). Data were reported as mean \pm standard deviation (SD) of at least three independent experiments. mRNA expression was presented as fold-change compared with the untreated control groups. Control group values were set at a fold-change equal to unity.

Inhibition of peripheral blood mononuclear cell (PBMC) proliferation by IDO-1- and COX-2-modified MSCs. Allogeneic PBMCs were isolated by Ficoll/Hypaque gradient centrifugation of peripheral venous blood (20-30 ml, that was collected from healthy volunteers). The preliminary tests showed that phytohemagglutinin (PHA; Sigma-Aldrich, St. Louis, MO, USA) stimulated lymphocyte proliferation at a final concentration of $10 \mu \mathrm{g} / \mathrm{ml}$. Additionally, the inhibitory effect of MSCs on lymphocyte proliferation was notable at a ratio of MSCs to lymphocytes of 1:10 to 1:20 (data not shown).

MSCs of the four groups were adapted to the co-culture medium (RPMI-1640 medium without phenol red, and supplemented with $10 \%$ FBS, 2 mM L-glutamine, $100 \mathrm{U} / \mathrm{ml}$ penicillin, and $100 \mu \mathrm{g} / \mathrm{ml}$ streptomycin) by gradient reduction of DMEM and plated in triplicate in 96-well microtiter plates. When MSCs achieved a $100 \%$ confluence, the cells were co-cultured with PHA-activated PBMCs. After three days, the suspended cells (mainly lymphocytes) from each well were transferred to a new 96-well plate, and then incubated with $10 \mu \mathrm{l}$ of Cell Counting kit-8 (CCK-8; Dojindo, Kumamoto, Japan). Absorbance at $450 \mathrm{~nm}$ was measured with a model 450 microplate reader (Bio-Rad Laboratories, Richmond, CA, USA). Experiments were performed in triplicate and were repeated at least twice.

Cytokine measurement in culture supernatant. After three days of co-culture of lymphocytes and MSCs, culture supernatants 
were collected to measure the levels of interferon- $\gamma($ IFN- $\gamma$ ) and tumor necrosis factor- $\alpha$ (TNF- $\alpha$ ) by enzyme-linked immunosorbent assay (ELISA) according to the manufacturer's instructions (R\&D Systems).

Cytotoxicity analysis. HeLa cells at a density of $3 \times 10^{4}$, were resuspended in RPMI-1640 medium (without phenol red), supplemented with 10\% FBS, and seeded in 96-well plates and used as target cells for cytotoxicity analysis. PHA-activated PBMCs were respectively co-cultured with MSCs from the four groups at a 1:10 ratio of MSCs to lymphocytes, respectively. After three days, suspended cells (mainly lymphocytes) from each group were transferred to HeLa cell pre-cultured 96-well plates with lymphocytes seeded at a density of $3 \times 10^{5}$ lymphocytes per well. The cells were incubated at $37^{\circ} \mathrm{C}$ in $5 \%$ $\mathrm{CO}_{2}$ in air for $6 \mathrm{~h}$. After this time period, $10 \mu \mathrm{l}$ of CCK-8 was added to the cultures and incubations were continued for an additional $1.5 \mathrm{~h}$. Relative absorbance values were measured at $450 \mathrm{~nm}$ as described above. Cytotoxic activity was calculated as: $\left[\mathrm{OD}_{(\mathrm{PBMC}+\mathrm{HeLa})}-\mathrm{OD}_{(\mathrm{PBMC})}\right] / \mathrm{OD}_{(\mathrm{HeL})}$.

Statistical analysis. Data are presented as the means \pm standard deviation. Data sets were compared via analysis of variance (ANOVA) followed by the Student's t-test for paired analysis of data sets between and within groups. Data were analyzed using SPSS software version 14.0 (SPSS Inc., Chicago, IL, USA). Differences between values were considered statistically significant at an $\alpha$-value of $\mathrm{P}<0.05$.

\section{Results}

Biological characteristics of UC-derived MSCs. After several passages, adherent cells from UC formed a monolayer of typical fibroblastic cells (Fig. 1A). Flow cytometric results showed that UC-derived cells shared most of their immunophenotype with MSCs, including a positive expression for stromal markers (CD29, CD44, CD73, CD90 and CD105), but a negative expression for the endothelial marker CD31, and the hematopoietic markers (CD34 and CD45) (Fig. 1B).

Construction of recombinant eukaryotic expression plasmids and electro-transfection. The amplification products of the $I D O-1$ and COX-2 genes (with relative size of 1,209 and $1,812 \mathrm{bp}$, respectively) were purified and digested with the restriction endonucleases $B g l \mathrm{II}$ and EcoRI to construct a recombinant plasmid by insertion into the pEGFP-N3 (4729 bp) vector. The result of rDNA sequence analysis confirmed the correct sequence and reading frame of IDO-1 (GenBank, NM_002164.5) and COX-2 (GenBank, NM_000963). MSCs were transfected with recombinant vectors with a transfection efficiency of $\sim 30 \%$ (Fig. $2 \mathrm{~A}$ and $\mathrm{B}$, as evaluated by observation of GFP fluorescence). The results of the western immunoblot analysis confirmed the existence of a thicker band for the IDO-1 and COX-2 proteins in the transfected MSCs as compared to MSCs without transfection, which demonstrated that the IDO-1 and COX-2 cDNA was expressed in MSCs (Fig. 2C).

Expression of immune-related genes in IDO-1- and COX-2modified MSCs. The results showed that the MSCs transfected

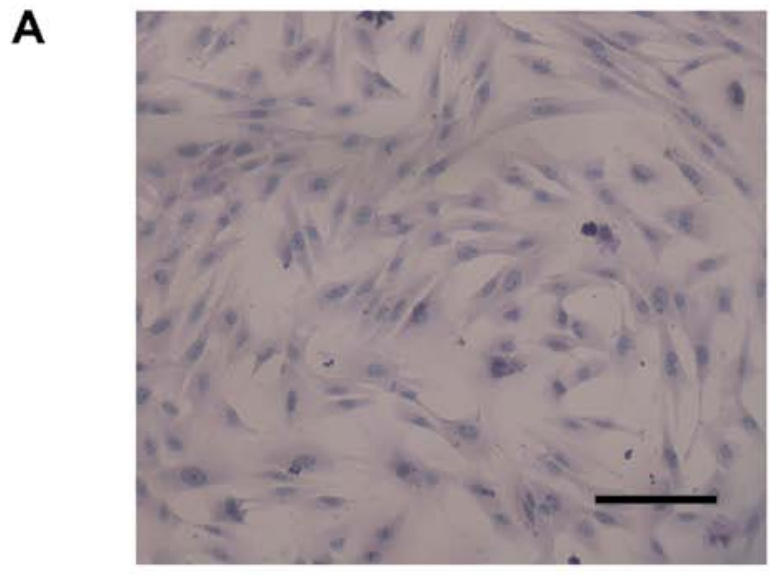

B
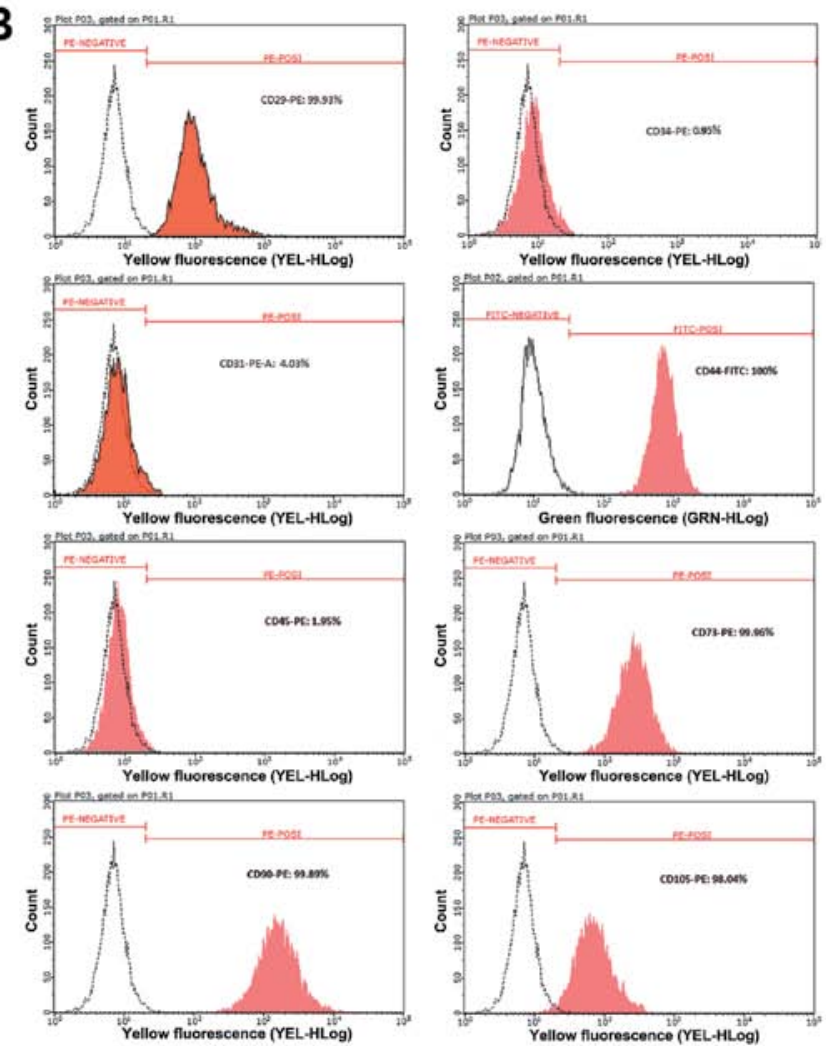

Figure 1. Morphological and immunophenotypic assessment of umbilical cord-derived mesenchymal stem cells. (A) Hematoxylin and eosin (H\&E) staining of umbilical cord-derived cells. These cells had a bipolar spindle shape and a monolayer pattern of growth. Scale bar, $100 \mu \mathrm{m}$. (B) Surface markers were detected by flow cytometric analysis. The cells expressed CD29, CD44, CD73, CD90 and CD105, but not CD31, CD34 or CD45.

with recombinant plasmids markedly upregulated the expression of the inserted genes (Fig. 3). Compared with the control group, the MSCs (IDO-1) group expressed very high levels of IDO-1, and downregulated other immunosupressive factors, such as COX-2, heme-oxygenase-1 (HO-1), inducible nitricoxide synthase (iNOS), TGF- $\beta$, IL-10, TNF- $\alpha$-stimulated gene/protein-6 (TSG-6) and human leukocyte antigen molecule 5 (HLA-G5). For the MSCs (COX-2) group, the expression levels of COX-2 and other immune regulatory genes were all elevated. However, apart from the expression of IDO-1 and 

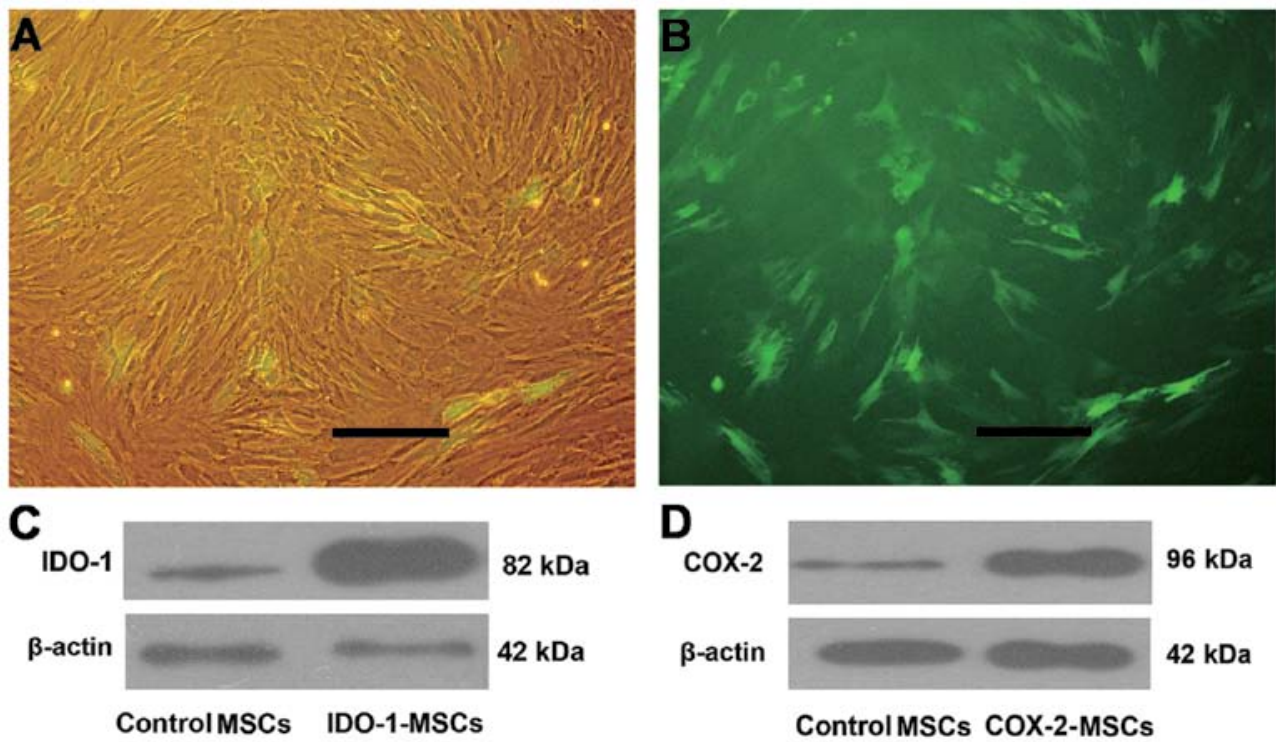

Figure 2. Electrical transfection of umbilical cord in mesenchymal stem cells (MSCs) shown as imaging and western blot analysis. (A and B) The expression of enhanced green fluorescent protein was observed by fluorescence microscopy after $48 \mathrm{~h}$, and the transfection efficiency was $\sim 30 \%$. Scale bar, $100 \mu \mathrm{m}$. (C) The expression of indoleamine 2,3-dioxygenase-1 (IDO-1) protein in the IDO-1 group of MSCs was higher than that in the untreated control MSCs. (D) The expression of cyclooxygenase-2 (COX-2) protein was more highly expressed in the COX-2 group of MSCs than the untransfected MSCs.

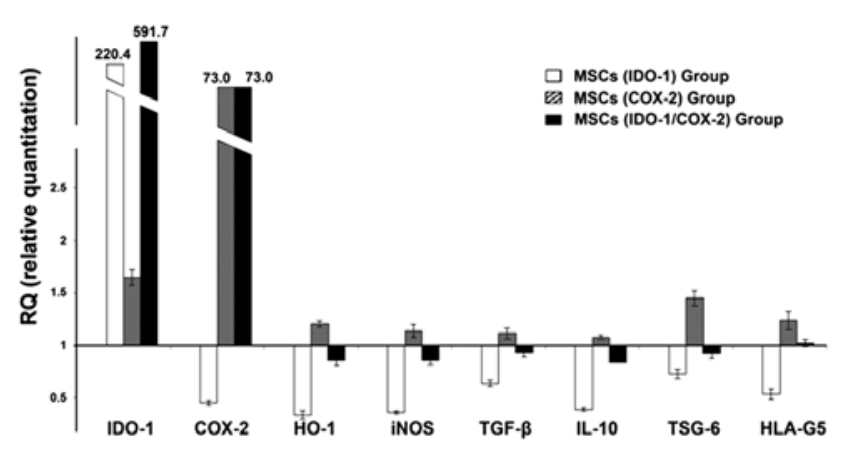

Figure 3. Immune-related gene expression in indoleamine 2,3-dioxygenase-1 (IDO-1)- and cyclooxygenase-2 (COX-2)-modified mesenchymal stem cells (MSCs). Immunomodulation-related gene expression was quantified by the reverse transcription-quantitative polymerase chain reaction (RT-qPCR) assay (the control group values were set at fold-change, 1). Compared with the untreated control group, the expression of IDO-1, COX-2, hemeoxygenase-1 (HO-1), inducible nitric-oxide synthase (iNOS), tumor necrosis factor- $\alpha$ (TNF- $\alpha$ ) stimulated gene/protein-6 (TSG-6), transforming growth factor- $\beta$ (TGF- $\beta$ ), human leukocyte antigen molecule 5 (HLA-G5) and interleukin-10 (IL-10) were enhanced in the COX-2 MSC group. However, apart from IDO-1 and COX-2, almost all the detected genes were dampened in the IDO-1 MSC group and the IDO-1/COX-2 MSC group.

COX-2, almost all the detected genes were dampened in the MSCs (IDO-1) and (IDO-1/COX-2) groups.

Potent immunosuppressive ability of COX-2-modified MSCs. In the preliminary experiments, PHA-stimulated lymphocytes showed significant proliferation from day 3 onwards compared to the untreated group (Fig. 4A). Thus, we collected the data from day 3 in subsequent co-culture assays. The results (Fig. 4B) showed that the inhibitory effect of MSCs on lymphocyte proliferation was statistically significant when transfected with IDO-1 or COX-2 compared with the control
MSCs. However, the inhibitory effect of MSCs (IDO-1/ COX-2) group was lower than that of the single-transfected MSCs (IDO-1) group or MSCs (COX-2) group.

Inflammatory cytokines expressed by PBMC, such as IFN- $\gamma$ and TNF- $\alpha$, were activated by PHA stimulation. However, following co-culture with MSCs, the expression levels of IFN- $\gamma$ and TNF- $\alpha$ were significantly reduced in PBMC. By contrast, the MSCs (COX-2) group more effectively inhibited the protein expression levels of IFN- $\gamma$ and TNF- $\alpha$ than their control group counterparts $(\mathrm{P}<0.05)$ (Fig. $5 \mathrm{~A}$ and $\mathrm{B})$.

In the cytotoxicity analysis, the results showed that the 10:1 ratio of lymphocytes relative to HeLa cells exerted a highly acceptable killing effect. By contrast, PHA-activated PBMC exhibited markedly increased cytotoxicity against HeLa cells. After co-culture with MSCs, the cytotoxic activity of PBMC was significantly lower than the non-transfected MSCs group $(\mathrm{P}<0.05)$. In addition, PBMC incubated with the MSCs (COX-2) group, had the lowest killing activity as compared with the non-transfected control group. However, the observations were not statistically different from each other (Fig. 5C).

\section{Discussion}

The immunosuppressive properties of MSCs potentially endow them with properties ideally suited as cellular products in the treatment of autoimmune and other immunemediated disorders $(29,30)$. Improving the immune regulatory efficiency of MSCs inevitably leads to improved clinical outcomes. As previously mentioned, IDO-1 and COX-2 play critical roles in the immunomodulation of MSCs $(23,24)$. In tryptophan catabolism, IDO-1 is a rate-limiting enzyme that inhibits antigen-specific T-cell proliferation and suppresses T-cell responses. In addition, COX-2 plays a key role in prostaglandin biosynthesis, which is helpful in PGE2-mediated immunosuppression (31-34). Therefore, in the current study, 



Figure 4. Lymphocyte proliferation assay of indoleamine 2,3-dioxygenase-1 (IDO-1)- and cyclooxygenase-2 (COX-2)-modified mesenchymal stem cells (MSCs). (A) Phytohemagglutinin (PHA)-primed lymphocytes showed significant proliferation from day 3 onwards as compared to the untreated group. (B) Proliferation of lymphocytes was suppressed by MSCs in the co-culture system. Overexpression of IDO-1 or COX-2 in MSCs promoted greater inhibitory functions as compared to their untreated MSC counterparts. However, no statistically significant differences were observed in the modified groups $\left({ }^{*} \mathrm{P}<0.05\right.$ and $\left.{ }^{* *} \mathrm{P}<0.01\right)$.
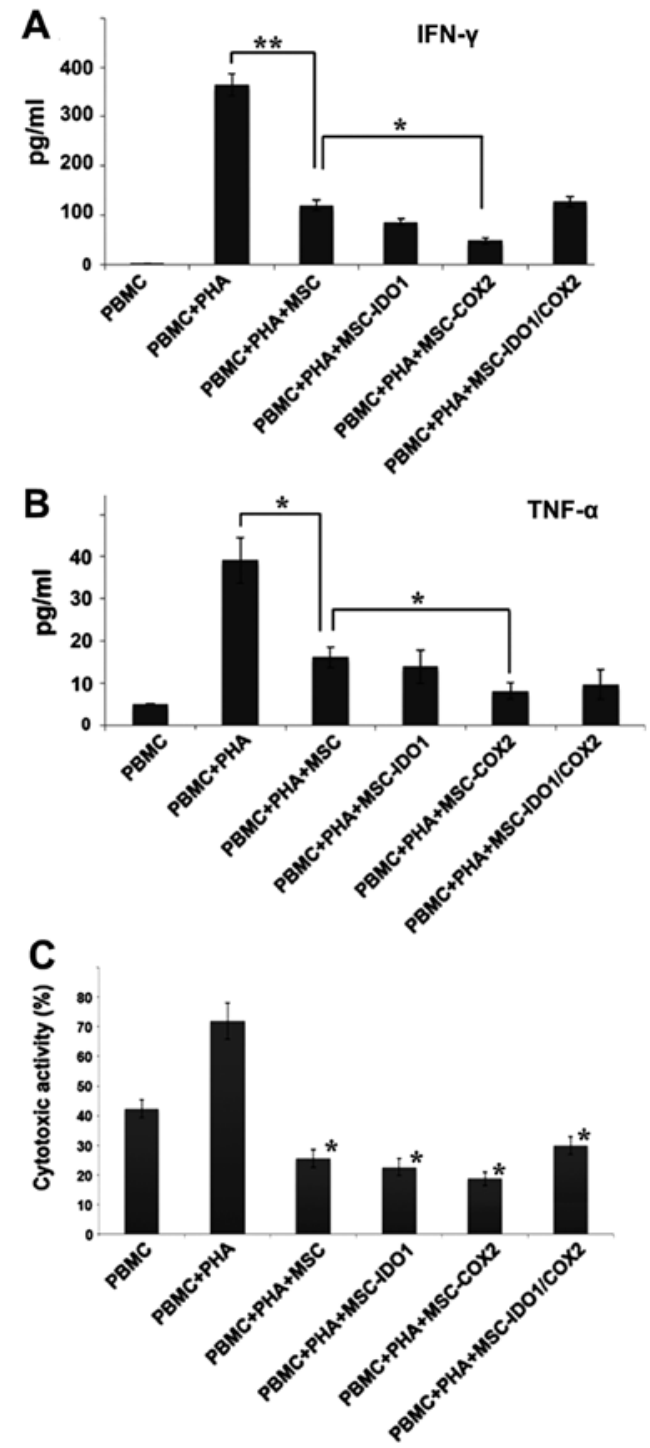

Figure 5. Reduction of lymphocyte activation by co-culture assay. (A and B) Enzyme-linked immunosorbent assay (ELISA) measurements show that the synthesis of interferon- $\gamma($ IFN- $\gamma)$ and tumor necrosis factor- $\alpha$ $(\mathrm{TNF}-\alpha)$ was significantly inhibited in peripheral blood mononuclear cel (PBMC) after co-culture with mesenchymal stem cells (MSCs), which was especially evident in the cyclooxygenase- $2(\mathrm{COX}-2)$ MSC group ( $\mathrm{P}<0.05$ and ${ }^{* *} \mathrm{P}<0.01$ ). Cytotoxicity assays showed that phytohemagglutinin (PHA)activated PBMC exhibited markedly increased cytotoxicity against HeLa cells. After co-culturing with MSCs, the cytotoxicity of PBMC for their targets was significantly reduced $(\mathrm{P}<0.05$, as compared with PBMNCs not cultured with MSCs). In addition, PBMC incubated with COX-2-transfected MSCs had the lowest killing activity as compared with the other groups. we attempted to improve the expression levels of IDO-1 or COX-2 to expand the active functional expression of key immune regulatory molecules that are needed in adoptive cellular immune therapy, thereby increasing the potential immune regulatory efficiency of MSCs.

Considering the clinical safety of the cell product, viral vectors are not the optimal choice. The short lifespan of allogeneic MSCs in recipient subjects also does not require a stable transfection (35-37). Thus, we reconstructed two recombinant plasmids to enhance the expression levels of IDO-1 and COX-2. The results of RT-qPCR and western immunoblotting confirmed the expression of IDO-1 and COX-2 in transfected MSCs. Further functional tests in co-culture assays, including lymphocyte proliferation and cytotoxicity assays, showed that COX-2-transfected MSCs exhibit more potent immunomodulatory properties than other groups. As shown by ELISA, the synthesis of IFN- $\gamma$ and TNF- $\alpha$ was significantly inhibited in PBMC after co-culture with COX-2-transfected MSCs. IFN- $\gamma$ is a cytokine that is produced primarily by T-lymphocytes and natural killer cells. IFN- $\gamma$ is critical for innate and adaptive immunity against viral and intracellular bacterial infections and for tumor control (38). It also alters the transcription of up to 30 genes that produce a variety of physiological and cell responses, including driving NK cell activity, increasing antigen presentation and lysosomal activity of macrophages. TNF- $\alpha$ was produced by macrophages, lymphoid cells, and fibroblasts, among other cells. TNF- $\alpha$ was originally characterized by its ability to induce tumor cell apoptosis and cachexia, and is now considered a central mediator of inflammation (39). Reduction in the levels of IFN- $\gamma$ and TNF- $\alpha$ dampens the occurrence of inflammation and immune rejection.

To elucidate the changes caused by transfection, we examined the expression of several immune regulatory genes in MSCs. In addition to $I D O-1$ and $C O X-2$, the expression of HO-1, iNOS, TSG-6, TGF- $\beta$, HLA-G5 and $I L-10$ were all increased after COX-2 transfection in MSCs. Previous findings have demonstrated that secretion of TGF- $\beta$ and induction of IL-10 contribute to the immunological dampening functions of MSC (40). MSC-released TSG-6 was also identified and shown to improve immunosuppression by interacting with CD44 on resident macrophages, which decreased TLR2/NFK-B signaling and thereby decreased the secretion of proinflammatory mediators $(41,42)$.

Another important molecule involved in MSC immune regulation involved the expression and secretion of HLA-G5. 
The soluble isoform of HLA-G5 that is secreted by MSCs is responsible for the suppression of T-cell proliferation, NK cell-mediated cytolysis, and IFN- $\gamma$ secretion (43). It was shown that iNOS is important in MSC immune suppression by limiting T-cell proliferation (44). The stress-inducible enzyme HO-1, which catalyzes the rate-limiting step of the degradation of heme to biliverdin, also has a suppressive effect on T-cell proliferation in human and rat MSCs $(45,46)$. Increased expression of these genes indicated that COX-2-enhanced MSCs were the most effective at enhancing immunosuppression, which is consistent with the results obtained from the functional tests.

The immunosuppressive effect of the MSCs (IDO-1) group was less than that of the MSCs (COX-2) group, suggesting the importance of PGE2 as compared to tryptophan/kynurenine among several immunomodulatory mechanisms. Notably, the immunosuppressive effect of MSCs with the two recombinant plasmids was weaker than MSCs transfected with only one recombinant plasmid. We hypothesize that the two plasmids may have competitive properties in the cell, resulting in inadequate translation. However, COX-2 transfection promoted positive outcomes, despite the instability of electrotransfection among different batches and cell membrane damage caused by the electrical pulses, which may have affected the efficacy of cell therapy. Additionally, the influence of COX-2 overexpression on cell viability remains to be investigated.

In conclusion, we obtained a novel population of MSCs that was derived following the overexpression of the immunerelated $C O X-2$ gene. The modified MSCs more potently inhibited the activation and proliferation of PBMCs. Thus, this gene-modified population of MSCs overexpressing COX-2, are expected to reduce the requirement of the number of infused cells, and improve the efficacy of treating autoimmune diseases and aGVHD.

\section{Acknowledgements}

The Major State Basic Research Development Program (2012CB966504), Shandong Province Natural Science Foundation (ZR2011HM007 and 2013GSF11812), and the Innovation Fund Project of Shandong University (grant no. 2012ZD023) all supported this study.

\section{References}

1. Johnson TV, Bull ND, Hunt DP, Marina N, Tomarev SI and Martin KR: Neuroprotective effects of intravitreal mesenchymal stem cell transplantation in experimental glaucoma. Invest Ophthalmol Vis Sci 51: 2051-2059, 2010.

2. Matsushita K, Morello F, Wu Y, et al: Mesenchymal stem cells differentiate into renin-producing juxtaglomerular (JG)-like cells under the control of liver X receptor-alpha. J Biol Chem 285: 11974-11982, 2010.

3. Williams AR and Hare JM: Mesenchymal stem cells: biology, pathophysiology, translational findings, and therapeutic implications for cardiac disease. Circ Res 109: 923-940, 2011.

4. Musumeci G, Lo Furno D, Loreto C, et al: Mesenchymal stem cells from adipose tissue which have been differentiated into chondrocytes in three-dimensional culture express lubricin. Exp Biol Med (Maywood) 236: 1333-1341, 2011.

5. Jurewicz M, Yang S, Augello A, et al: Congenic mesenchymal stem cell therapy reverses hyperglycemia in experimental type 1 diabetes. Diabetes 59: 3139-3147, 2010.
6. Curley GF, Hayes M, Ansari B, et al: Mesenchymal stem cells enhance recovery and repair following ventilator-induced lung injury in the rat. Thorax 67: 496-501, 2012.

7. Li J, Li D, Liu X, Tang S and Wei F: Human umbilical cord mesenchymal stem cells reduce systemic inflammation and attenuate LPS-induced acute lung injury in rats. J Inflamm (Lond) 9: 33, 2012.

8. Hu KX, Sun QY, Guo M and Ai HS: The radiation protection and therapy effects of mesenchymal stem cells in mice with acute radiation injury. Br J Radiol 83: 52-58, 2010.

9. Zoja C, Garcia PB, Rota C, et al: Mesenchymal stem cell therapy promotes renal repair by limiting glomerular podocyte and progenitor cell dysfunction in adriamycin-induced nephropathy. Am J Physiol Renal Physiol 303: F1370-F1381, 2012.

10. Nauta AJ and Fibbe WE: Immunomodulatory properties of mesenchymal stromal cells. Blood 110: 3499-3506, 2007.

11. Aggarwal S and Pittenger MF: Human mesenchymal stem cells modulate allogeneic immune cell responses. Blood 105: 1815-1822, 2005

12. Atoui R, Shum-Tim D and Chiu RC: Myocardial regenerative therapy: immunologic basis for the potential 'universal donor cells'. Ann Thorac Surg 86: 327-334, 2008.

13. Rice CM, Kemp K, Wilkins A and Scolding NJ: Cell therapy for multiple sclerosis: an evolving concept with implications for other neurodegenerative diseases. Lancet 382: 1204-1213, 2013.

14. Kim N, Im KI, Lim JY, et al: Mesenchymal stem cells for the treatment and prevention of graft-versus-host disease: experiments and practice. Ann Hematol 92: 1295-1308, 2013.

15. Ning H, Yang F, Jiang M, et al: The correlation between cotransplantation of mesenchymal stem cells and higher recurrence rate in hematologic malignancy patients: outcome of a pilot clinical study. Leukemia 22: 593-599, 2008.

16. Ball LM, Bernardo ME, Roelofs H, et al: Cotransplantation of ex vivo expanded mesenchymal stem cells accelerates lymphocyte recovery and may reduce the risk of graft failure in haploidentical hematopoietic stem-cell transplantation. Blood 110: 2764-2767, 2007.

17. Lazarus HM, Koc ON, Devine SM, et al: Cotransplantation of HLA-identical sibling culture-expanded mesenchymal stem cells and hematopoietic stem cells in hematologic malignancy patients. Biol Blood Marrow Transplant 11: 389-398, 2005.

18. Koç ON, Gerson SL, Cooper BW, et al: Rapid hematopoietic recovery after coinfusion of autologous-blood stem cells and culture-expanded marrow mesenchymal stem cells in advanced breast cancer patients receiving high-dose chemotherapy. J Clin Oncol 18: 307-316, 2000.

19. Le Blanc K, Samuelsson H, Gustafsson B, et al: Transplantation of mesenchymal stem cells to enhance engraftment of hematopoietic stem cells. Leukemia 21: 1733-1738, 2007.

20. Ji JF, He BP, Dheen ST and Tay SS: Interactions of chemokines and chemokine receptors mediate the migration of mesenchymal stem cells to the impaired site in the brain after hypoglossal nerve injury. Stem Cells 22: 415-427, 2004.

21. Devine SM, Cobbs C, Jennings M, Bartholomew A and Hoffman R: Mesenchymal stem cells distribute to a wide range of tissues following systemic infusion into nonhuman primates. Blood 101: 2999-3001, 2003.

22. Jung JW, Kwon M, Choi JC, et al: Familial occurrence of pulmonary embolism after intravenous, adipose tissue-derived stem cell therapy. Yonsei Med J 54: 1293-1296, 2013.

23. Spaggiari GM, Abdelrazik H, Becchetti F and Moretta L: MSCs inhibit monocyte-derived DC maturation and function by selectively interfering with the generation of immature DCs: central role of MSC-derived prostaglandin E2. Blood 113: 6576-6583, 2009.

24. Opitz CA, Litzenburger UM, Lutz C, et al: Toll-like receptor engagement enhances the immunosuppressive properties of human bone marrow-derived mesenchymal stem cells by inducing indoleamine-2,3-dioxygenase-1 via interferon-beta and protein kinase R. Stem Cells 27: 909-919, 2009.

25. Sato K, Ozaki K, Oh I, et al: Nitric oxide plays a critical role in suppression of T-cell proliferation by mesenchymal stem cells. Blood 109: 228-234, 2007.

26. Yoo SW, Chang DY, Lee HS, et al: Immune following suppression mesenchymal stem cell transplantation in the ischemic brain is mediated by TGF- $\beta$. Neurobiol Dis 58: 249-257, 2013.

27. Djouad F, Charbonnier LM, Bouffi C, et al: Mesenchymal stem cells inhibit the differentiation of dendritic cells through an interleukin-6-dependent mechanism. Stem Cells 25: 2025-2032, 2007. 
28. Németh K, Leelahavanichkul A, Yuen PS, et al: Bone marrow stromal cells attenuate sepsis via prostaglandin E(2)-dependent reprogramming of host macrophages to increase their interleukin-10 production. Nat Med 15: 42-49, 2009.

29. Sui W, Hou X, Che W, et al: Hematopoietic and mesenchymal stem cell transplantation for severe and refractory systemic lupus erythematosus. Clin Immunol 148: 186-197, 2013.

30. Luz-Crawford P, Kurte M, Bravo-Alegria J, et al: Mesenchymal stem cells generate a $\mathrm{CD} 4^{+} \mathrm{CD} 25^{+} \mathrm{Foxp} 3^{+}$regulatory $\mathrm{T}$ cell population during the differentiation process of Th1 and Th17 cells Stem Cell Res Ther 4: 65, 2013.

31. Spaggiari GM, Capobianco A, Abdelrazik H, Becchetti F, Mingari MC and Moretta L: Mesenchymal stem cells inhibit natural killer-cell proliferation, cytotoxicity, and cytokine production: role of indoleamine 2,3-dioxygenase and prostaglandin E2. Blood 111: 1327-1333, 2008.

32. English K, Ryan JM, Tobin L, Murphy MJ, Barry FP and Mahon BP: Cell contact, prostaglandin E(2) and transforming growth factor beta 1 play non-redundant roles in human mesenchymal stem cell induction of $\mathrm{CD} 4{ }^{+} \mathrm{CD} 25$ (High) forkhead box $\mathrm{P}^{+}{ }^{+}$regulatory T cells. Clin Exp Immunol 156: 149-160, 2009.

33. Duffy MM, Pindjakova J, Hanley SA, et al: Mesenchymal stem cell inhibition of T-helper 17 cell- differentiation is triggered by cell-cell contact and mediated by prostaglandin E2 via the EP4 receptor. Eur J Immunol 41: 2840-2851, 2011.

34. Yañez R, Oviedo A, Aldea M, Bueren JA and Lamana ML: Prostaglandin E2 plays a key role in the immunosuppressive properties of adipose and bone marrow tissue-derived mesenchymal stromal cells. Exp Cell Res 316: 3109-3123, 2010.

35. Pittenger MF and Martin BJ: Mesenchymal stem cells and their potential as cardiac therapeutics. Circ Res 95: 9-20, 2004.

36. Tamama K, Kawasaki H and Wells A: Epidermal growth factor (EGF) treatment on multipotential stromal cells (MSCs). Possible enhancement of therapeutic potential of MSC. J Biomed Biotechnol 2010: 795385, 2010.
37. Freyman T, Polin G, Osman H, et al: A quantitative, randomized study evaluating three methods of mesenchymal stem cell delivery following myocardial infarction. Eur Heart J 27: 1114-1122, 2006.

38. Schroder K, Hertzog PJ, Ravasi T and Hume DA: Interferongamma: an overview of signals, mechanisms and functions. J Leukoc Biol 75: 163-189, 2004.

39. Pfeffer K: Biological functions of tumor necrosis factor cytokines and their receptors. Cytokine Growth Factor Rev 14: 185-191, 2003.

40. Liu H, Lu K, MacAry PA, et al: Soluble molecules are key in maintaining the immunomodulatory activity of murine mesenchymal stromal cells. J Cell Sci 125: 200-208, 2012.

41. Qi Y, Jiang D, Sindrilaru A, et al: TSG-6 released from intradermally injected mesenchymal stem cells accelerates wound healing and reduces tissue fibrosis in murine full-thickness skin wounds. J Invest Dermatol 134: 526-537, 2014.

42. Prockop DJ: Concise review: two negative feedback loops place mesenchymal stem/stromal cells at the center of early regulators of inflammation. Stem Cells 31: 2042-2046, 2013.

43. Selmani Z, Naji A, Zidi I, et al: Human leukocyte antigen-G5 secretion by human mesenchymal stem cells is required to suppress $\mathrm{T}$ lymphocyte and natural killer function and to induce $\mathrm{CD} 4{ }^{+} \mathrm{CD} 25^{\text {high }} \mathrm{FOXP}^{+}$regulatory T cells. Stem Cells 26: 212-222, 2008.

44. Ren G, Zhang L, Zhao X, et al: Mesenchymal stem cell-mediated immunosuppression occurs via concerted action of chemokines and nitric oxide. Cell Stem Cell 2: 141-150, 2008.

45. Chabannes D, Hill M, Merieau E, et al: A role for heme oxygenase- 1 in the immunosuppressive effect of adult rat and human mesenchymal stem cells. Blood 110: 3691-3694, 2007.

46. Brusko TM, Wasserfall CH, Agarwal A, Kapturczak MH and Atkinson MA: An integral role for heme oxygenase-1 and carbon monoxide in maintaining peripheral tolerance by $\mathrm{CD} 4^{+} \mathrm{CD} 25^{+}$ regulatory T cells. J Immunol 174: 5181-5186, 2005. 